\title{
THE ONE-DIMENSIONAL FRACTIONAL QUANTUM OPERATOR OF MOMENTUM
}

\author{
P. Miškinis \\ Department of Physics, Vilnius Gediminas Technical University, Sauletekio 11, LT-10223 Vilnius, Lithuania \\ E-mail: paulius.miskinis@fm.vtu.lt
}

Received 7 April 2006

\begin{abstract}
In the case of the quantum generalization of Lévy processes, expressions for the Hermitian operator of momentum and its eigenfunctions are proposed. The normalization constant has been determined and its relation to the translation operator is shown. The interrelation between the momentum and the wave number has been generalized for the processes with a non-integer dimensionality $\alpha$.
\end{abstract}

Keywords: Lévy processes, quantum mechanics, fractional calculus

PACS: 5.40.Fb, 05.30.Pr, 03.65.Db, 03.65.Sq

\section{Introduction}

The physical basis for the existence of quantum mechanics comprises a series of phenomena described by the mathematical theory of the Wienerian processes.

The Lévy stochastic process is a natural generalization of the Brownian motion or the Wiener stochastic process [1]. The foundation for this generalization is the theory of stable probability distributions developed by Lévy [2]. The most fundamental property of the Lévy distributions is the stability in respect to addition, in accordance with the generalized central limit theorem. Thus, from the probability theory point of view, the stable probability law is a generalization of the well-known Gaussian law. The Lévy processes are characterized by the Lévy index $\alpha$, which takes values $0<\alpha \leq 2$. At $\alpha=2$ we have the Gaussian process or the process of the Brownian motion. Lévy process is widely used to model a variety processes such as anomalous diffusion [3], turbulence [4], chaotic dynamics [5], plasma physics [6], financial dynamics [7], biology and physiology [8] (for recent references see e. g. [9-11]) .

The constantly increasing number of experimental facts in various fields of knowledge related to classical non-Wienerian processes evokes a natural desire to "close" the commutative diagram shown in Fig. 1 and, at least formally, to consider the possible existence of a quantum analogue of a narrower class of phenomena related to Lévy processes, the so-called fractional

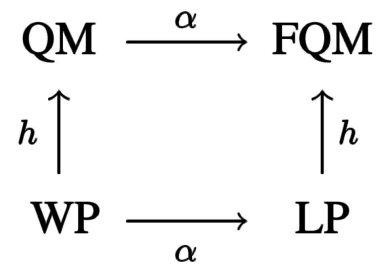

Fig. 1. Schematic representation of interrelations of Wienerian processes (WP), Lévy processes (LP), quantum mechanics (QM), and fractional quantum mechanics (FQM).

quantum mechanics (FQM) [12-14]. Unfortunately, these works are not aimed at a thorough analysis of the properties of the quantum operator of momentum. The lack of such analysis results in some inaccuracies of even gross blunders while formulating FQM (see Conclusions).

The present note offers a brief discussion of one of the crucial issues related to FQM, which is the onedimensional operator of momentum.

Like in usual quantum mechanics (QM), one-dimensional problems are a kind of excess idealization. Nevertheless, they may be used for elucidating the fundamental features of FQM. One-dimensional problems arise while considering the three-dimensional evolutionary equation in which the interaction potential depends on a single coordinate. This fact allows, with the aid of a corresponding factorization, to move to a simpler one-dimensional evolutionry equation.

The purpose of this paper is formulation, in the explicit form, of a quantum expression of the one- 
dimensional operator of momentum for the fractional probability processes.

\section{Fractional quantum operator of momentum}

The classical definition of momentum in QM follows from the invariance of the Hamiltonian of the quantum system $\hat{H}$ with respect to the infinitesimal displacements $\delta x$. Under such transformation, the wave function $\psi(x)$ turns into the function

$$
\psi(x+\delta x)=\psi(x)+\delta x \partial_{x} \psi=\left(1+\delta x \partial_{x}\right) \psi(x),
$$

here $\partial_{x}$ is the differentiation operator over the space variable $x[15,16]$.

However, it may turn out that $\partial_{x} \psi$ does not exist, but there exists the so-called fractional derivative $\partial_{x}^{\alpha} \psi$ in which the order of the derivative $\alpha$ may be both an integer and a fractional number. For the function determined on the whole real axis $\mathbb{R}$, the right and left derivatives of the order $\alpha$ are defined as

$$
\begin{aligned}
\partial_{ \pm}^{\alpha} \psi(x)= & \frac{\{\alpha\}}{\Gamma(1-\{\alpha\})} \\
& \times \int_{0}^{\infty} \frac{\psi^{([\alpha])}(x)-\psi^{([\alpha])}(x \mp \xi)}{\xi^{1+\{\alpha\}}} \mathrm{d} \xi,
\end{aligned}
$$

where $[\alpha]$ and $\{\alpha\}$ are the integer and the fractional parts of the parameter $\alpha$ (for details, see Appendix). For the bilateral derivatives to exist, it is sufficient that $\psi(x) \in C^{[\alpha]}(\Omega)$, where $C^{[\alpha]}(\Omega)$ is a set of continuously differentiated functions of the order $[\alpha]$ determined on the domain $\Omega$ [10].

Another peculiarity related to the operator of momentum is the expansion of the wave function $\psi(x)$ into a Taylor series by fractional powers [11]

$$
\psi(x)=\sum_{n=0}^{[\alpha]} c_{n}^{(\alpha)}\left(x-x_{0}\right)^{\alpha+n}+R_{n}(x),
$$

where $c_{n}^{(\alpha)}$ are numerical coefficients and $R_{n}(x)$ is the residual term, which provides a better approach to the initial function. In all such cases, determination of the quantum operator of momentum should be specified.

It is reasonable to suppose that the momentum operator should be proportional to the fractional derivative:

$$
\hat{p}=C \partial_{+}^{\alpha} \psi(x),
$$

here $C$ is a certain coefficient of proportionality. For $\alpha \rightarrow 0$, we must obtain a usual quantum operator of momentum, $\hat{p}=-\mathrm{i} \hbar \partial_{x}$. Thus, in FMQ we always deal with two kinds of limit transitions: (i) $\hbar \rightarrow 0$, when we shift to classical mechanics, and (ii) $\alpha \rightarrow 0$, when we turn to usual QM (see Fig. 1).

The kind of the coefficient $C$ in the expression for the momentum (4) is best defined if on the whole real axis we consider a plane wave of the form

$$
\psi(x, t)=A \mathrm{e}^{\mathrm{i} \kappa x-\mathrm{i} E / \hbar t}, \kappa=\left(\frac{p}{\hbar l_{0}^{\alpha-1}}\right)^{1 / \alpha},
$$

where $\hbar$ is the Planck constant and $l_{0}$ is a certain peculiar scale of the length of the nonlocal process under consideration.

Let us impose a requirement for the momentum operator (4) to obey the eigenvalue equation $\hat{p} \psi=p \psi$. Applying the property of the fractional derivatives, $\partial_{+}^{\alpha} \mathrm{e}^{\kappa x}=\kappa^{\alpha} \mathrm{e}^{\kappa x}(\operatorname{Re} \kappa>0)($ see Appendix), we obtain that

$$
C=(-\mathrm{i})^{\alpha} \hbar l_{0}^{\alpha-1} .
$$

For the values observed in QM to be real, the corresponding operators should be Hermitian. It is easy to see that the quantum operator of momentum (4) with the constant $C$ from (6) is non-Hermitian. In order to obtain a Hermitian operator of momentum, to the type (4) operator we will add a Hermite-conjugated operator $\hat{p}^{+}$; then, the momentum operator determined in this way

$$
\hat{p}=\frac{\hbar l_{0}^{\alpha-1}}{2}\left[\left(-\mathrm{i} \partial_{+}\right)^{\alpha}+\left(\mathrm{i} \tilde{\partial}_{-}\right)^{\alpha}\right],
$$

here $\sim$ is the symbol of transposition, will be clearly Hermitian. Indeed, the momentum operator (7) $\hat{p}=$ $\left(\hat{p}_{+}+\hat{p}_{-}\right) / 2$ will be Hermitian because of the idempotency of the operation of Hermitian conjugation $\left(\left(\hat{p}^{+}\right)^{+}=\hat{p}\right)$ and the structure of the operator itself $\left(\hat{p}_{-}=\left(\hat{p}_{+}\right)^{+}\right)$. On the other hand, employing the rule of fractional integration by parts (see Appendix),

$$
\begin{aligned}
p^{*}= & \int_{-\infty}^{+\infty} \varphi^{*} \hat{p}^{+} \psi \mathrm{d} x= \\
& \frac{1}{2} \int_{-\infty}^{+\infty} \varphi^{*}\left(\hat{p}_{-}+\hat{p}_{+}\right) \psi \mathrm{d} x=p,
\end{aligned}
$$

we directly see that the momentum operator is Hermitian for the different functions of state $\varphi$ and $\psi$.

Thus, we obtain that the operator (7) is Hermitian and its eigenvalues on the whole real axis are plane waves of the (5) type. Like in the classical case, the eigenvalues of the momentum operator do not belong 
to the class $L^{2}(\mathbb{R})$. Therefore, they don't describe the physically realizable states of the quantum particle. These eigenfunctions should be regarded as the basic functions, which comprise the complete system of functions.

\section{Wave function normalization}

To determine the constant normalization $A$ in the expression for the plane wave (5), we will take that

$$
\int \psi_{p}^{*} \psi_{0} \mathrm{~d} x=\delta(p)
$$

This is a particular case of the conventional condition $\int \psi_{p^{\prime}}^{*} \psi_{p} \mathrm{~d} x=\delta\left(p^{\prime}-p\right)$ for $p \equiv 0$. Using the property of the $\delta$-function, we shall obtain that

$$
A=\sqrt{\frac{|\kappa|}{2 \pi|p|}}, \quad \kappa \equiv\left(\frac{p}{\hbar l_{0}^{\alpha-1}}\right)^{1 / \alpha} .
$$

Let us specify the peculiarities of such normalization. Firstly, generally speaking, the amplitude is a complex magnitude; secondly, it depends on the eigenvalue of the momentum $p$. Only when $\alpha \rightarrow 1$, as the case should be, $A \rightarrow 1 / \sqrt{2 \pi \hbar}$.

Inasmuch as the physical sense applies not to the amplitude itself but to $|A|^{2}$, the complex nature of $A$ does not contradict unitarity. However, because of the complex nature of the amplitude we may get an impression that we deal with a damping wave; however, actually there is no damping, because $A \neq A(x, t)$. Besides, the same conclusion results from analysis of the dispersion expression of the corresponding Hamiltonian. The dependence $A=A(p)$ is not a matter of principle and may be avoided by a suitable choice of the normalization condition. For instance, under condition

$$
\int \psi_{\kappa^{\prime}}^{*} \psi_{\kappa} \mathrm{d} x=\delta\left(\kappa^{\prime}-\kappa\right)
$$

the dependence $A(p)$ is absent.

Another important circumstance should be noted as regards the type of the momentum operator. Transition to momentum representation is not a Fourier transformation. Momentum representation should be understood in the sense of $f$-representation:

$$
\psi(x)=\int a_{f} \psi_{f}(x) \mathrm{d} f, \quad \int \psi_{f^{\prime}}^{*} \psi_{f} \mathrm{~d} x=\delta\left(f^{\prime}-f\right) .
$$

\section{Translation operator}

Lastly, let us derive the formula to express, through the momentum operator $\hat{p}$, the parallel translation operator in space to any finite (not only infinitesimal) distance. From the definition of such an operator it follows:

$$
\hat{T}_{a+}^{\alpha} \psi(x)=\psi(x-a), \quad \hat{T}_{b-}^{\alpha} \psi(x)=\psi(x+b) .
$$

In this case, $a$ and $b$ denote the values of finite displacements but not the coordinate ends of the interval.

Expanding the function $\psi(x-a)$ in the neighbourhood of the point $x$ into a Taylor series by fractional powers as in (3) and employing the expression for the "right-hand" and "left-hand" parts of the momentum operator,

$$
\hat{p}_{+}^{\alpha}=-\mathrm{i} \hbar l_{0}^{\alpha-1} \partial_{+}^{\alpha}, \quad \hat{p}_{-}^{\alpha}=\mathrm{i} \hbar l_{0}^{\alpha-1} \tilde{\partial}_{-}^{\alpha},
$$

we obtain that

$$
\begin{aligned}
\hat{T}_{a+}^{\alpha}= & \sum_{k=0}^{\infty} \frac{(\mathrm{i} a)^{\alpha+k-1}}{\left(\hbar l_{0}^{\alpha-1}\right)^{(\alpha+k-1) / \alpha}} \frac{\hat{p}_{+}^{(\alpha+k-1)}}{\Gamma(\alpha+k)} \equiv \\
& \mathrm{E}_{1-\alpha}^{\kappa_{a} \hat{p}_{+}^{\alpha}},
\end{aligned}
$$

where $\mathrm{E}_{\mu}^{z}$ is the generalized exponential function (see Appendix). These are exactly the finite displacement operators we have been searching for.

For $\alpha \rightarrow 1$, for $\hat{T}_{a+}^{\alpha}$ we obtain that

$$
\hat{T}_{a+}^{\alpha} \rightarrow \sum_{k=0}^{\infty}\left(\frac{\mathrm{i} a}{\hbar}\right)^{k} \frac{\hat{p}_{+}^{k}}{k !} \equiv \mathrm{e}^{\mathrm{i} a / \hbar \hat{p}_{+}} .
$$

The expression $\hat{T}_{b-}^{\alpha}$ could be obtain by substituting in equation (16) $a \rightarrow-b$ and $\hat{p}_{+}^{\alpha} \rightarrow \hat{p}_{-}^{\alpha}$.

\section{Conclusions}

Note that the classical restriction on the smoothness of the wave function $\psi(x) \in C^{2}([a, b])$ does not hold here. The restriction on $\psi(x)$ follows from the continuity equation; however, in the case of fractional dimension we can show that the condition of continuity is changed, and the limitation on $\psi(x)$ is reduced to $\psi(x) \in C^{[\alpha]}([a, b])$.

Another note pertains to the structure of the momentum operator. It seems highly significant that the momentum operator consists of two parts - the right and left displacements. In classical fractional mechanics, it is quite possible to limit ourselves to one of these two 
components, $\hat{p}_{+}$or $\hat{p}_{-}$. In the quantum case it is impossible, because the full operator of momentum is a Hermitian one.

The limit transition $\hbar \rightarrow 0$ for $\{\alpha\} \neq 0$ means transition to classical fractional mechanics. However, the form of the momentum operator undergoes no qualitative change: $\hat{p}=\left(\hat{p}_{+}+\hat{p}_{-}\right) / 2$, i. e. it consists of two parts, each being proportional to its one-sided derivative. For linear evolutionary equations of classical (not quantum) fractional mechanics this type of structure of the momentum operator may be simplified if $\hat{p}=\hat{p}_{+}$or $\hat{p}=\hat{p}_{-}$. However, here additional considerations are necessary. For the nonlinear fractional evolutionary processes it is impossible in principle, because $\hat{p}_{+}=\hat{p}_{-}$is the condition of smoothness.

Note here, too, that all results for the Hermitian operator of momentum are valid in the case of the Riesz quantum derivative: ${ }^{R} D_{x}^{\alpha} \phi(x) \propto\left[(-\mathrm{i} \partial)_{+}^{\alpha}+\right.$ $\left.(\mathrm{i} \tilde{\partial})_{-}^{\alpha}\right] \phi(x)$.

From the definition of $\kappa$ there follows an interrelation between the momentum and the wave number:

$$
p=\hbar l_{0}^{\alpha-1} \kappa^{\alpha},
$$

so for $\alpha \rightarrow 1$ we have $\kappa \rightarrow k$, and the expression (17) turns into $p=\hbar k$.

The appearance of the characteristic length scale of $l_{0}$ and the power dependence of the quantum particle momentum on the wave number directly indicate the fractional character of quantum mechanics.

Thus we have the Hermitian quantum operator of momentum (7) with the eigenfunctions (5). This allows us to construct the quadratic form of the Hamiltonian $\hat{H} \propto \hat{p}^{2}$ instead of the power form $\hat{H} \propto D_{\alpha}|p|^{\alpha}$, and the Hermitian Hamiltonian instead of non-Hermitian proposed in $[12,13]$, and the unitarian Hamiltonian instead of non-unitarian proposed in [14]. However, the Hamiltonian of the FQM is the subject of a special investigation and of the forthcoming paper.

\section{Appendix}

The right and left fractional derivatives are defined in the form

$$
\begin{aligned}
\partial_{ \pm}^{\alpha} f(x)= & \frac{\{\alpha\}}{\Gamma(1-\{\alpha\})} \\
& \times \int_{0}^{+\infty} \frac{f^{[\alpha]}(x)-f^{[\alpha]}(x \mp \xi)}{\xi^{1+\{\alpha\}}} \mathrm{d} \xi
\end{aligned}
$$

where $\Gamma(z)$ is the Euler $\Gamma$-function, $\alpha=[\alpha]+\{\alpha\}$ is the sum of the integer and the fractional parts of the real number $\alpha \in \mathbb{R}$. These are the so-called Marchaud derivatives [17], which on the whole real axis are more natural than, e. g., the Riemann-Liouville derivatives. For instance, for the functions determined on $\mathbb{R}: f(x) \in L^{p}$, where $1 \leq p<1 / \alpha$,

$$
\partial_{ \pm}^{\alpha} I_{ \pm}^{\alpha} f(x)=f(x)
$$

here $I_{ \pm}^{\alpha} f(x)$ is a fractional integral of the order $\alpha$, whereas for the Riemann-Liouville derivatives the property (A2) holds only if $\alpha=1$. The Marchaud derivatives, on sufficiently "good" functions, coincide with the Riemann-Liouville derivatives, however, in contrast to the latter, they allow even an increase of the functions of the order below $\alpha$ at the infinity. The differences between the Riemann-Liouville and Marchaud functions, related to the behaviour at the infinity, are absent in the case of a finite interval [18].

Let us remind here that the one-parametrical family of linear limited operators $\left\{T_{\alpha}\right\}, \alpha \geq 0$ in the Banach space $X$ comprises a semi-group, if

$$
\begin{aligned}
& T_{\alpha} T_{\beta}=T_{\alpha+\beta}, \quad \alpha \geq 0, \beta \geq 0, \\
& T_{0} \varphi=\varphi, \quad \forall \varphi \in X .
\end{aligned}
$$

A semigroup of operators is called strongly continuous if

$$
\begin{aligned}
& \lim _{\alpha \rightarrow \alpha_{0}}\left\|T_{\alpha} \varphi-T_{\alpha_{0}} \varphi\right\|=0, \\
& 0 \leq \alpha_{0}<\infty, \quad \forall \varphi \in X .
\end{aligned}
$$

From the semigroup character of the family $\left\{\partial_{ \pm}^{\alpha}\right\}$ (A1) it follows that if a semigroup is strongly continuous when $\alpha=0$, it is inevitably strongly continuous for all $\alpha \geq 0$.

From the definition of the fractional operators it follows that fractional integration operators comprise in $L^{p}(a, b), p \geq 1$ a semigroup, which is continuous in the uniform topology for all $\alpha>0 . L^{p}(a, b)$, as usual, denote a set of functions $|f|^{p}$ in the $p$ power, which are measurable according to Lebesgue.

The form of the approximation of the operator $I_{a+}^{\alpha}$ to unity when $\alpha \rightarrow 0$ is conditioned by the form of the generating operator $\mathcal{L}(x) \equiv \lim _{\alpha \rightarrow+0}\left\|I_{a+}^{\alpha} \varphi-\varphi\right\| / \alpha$. The expression for the operator is calculated from the definition using the L'Hôpital rule. The calculation 
gives the following expression for the form of $I_{a+}^{\alpha} \varphi$ approximation:

$$
\mathcal{L}(x)=\frac{\mathrm{d}}{\mathrm{d} x} \int_{a}^{x} \ln (x-t) \varphi(x) \mathrm{d} x-\Gamma^{\prime}(1) \varphi(x),(
$$

for almost all $x$.

It is convenient to make use of unified designation also for the other integrals and derivatives, considering that

$$
\partial_{+}^{\alpha} f=I_{+}^{-\alpha} f=\left(I_{+}^{\alpha}\right) f, \alpha>0 .
$$

The semigroup character of the fractional derivatives is

$$
\partial_{ \pm}^{\alpha} \partial_{ \pm}^{\beta} f(x)=\partial_{ \pm}^{\beta} \partial_{ \pm}^{\alpha} f(x)=\partial_{ \pm}^{\alpha+\beta} f(x) .
$$

The fractional integration by parts

$$
\int_{a}^{b} \varphi(x) \partial_{+}^{\alpha} \psi(x) \mathrm{d} x=\int_{a}^{b} \psi(x) \partial_{-}^{\alpha} \varphi(x) \mathrm{d} x .
$$

Example 1a:

$$
\partial_{+}^{\alpha} \mathrm{e}^{\lambda x+\mu}=\lambda^{\alpha} \mathrm{e}^{\lambda x+\mu}(\operatorname{Re} \lambda>0) .
$$

Example 1b:

$$
\partial_{-}^{\alpha} \mathrm{e}^{\lambda x+\mu}=(-\lambda)^{\alpha} \mathrm{e}^{\lambda x+\mu}(\operatorname{Re} \lambda<0) .
$$

Some special functions are a very convenient tool for applications, e.g., the Mittag-Leffler function:

$$
\begin{aligned}
& \mathrm{E}_{\alpha, \beta}(z)=\sum_{k=0}^{\infty} \frac{z^{k}}{\Gamma(\alpha k+\beta)}, \\
& \alpha>0, \beta>0 .
\end{aligned}
$$

It is obvious that $\mathrm{E}_{1,1}(z)=\mathrm{e}^{z}$. tion:

Another example is the fractional exponential func-

$$
\begin{aligned}
\mathrm{E}_{\mu}^{z} & =\sum_{k=0}^{\infty} \frac{z^{k-\mu}}{\Gamma(k-\mu+1)}=\mathrm{e}^{-\mu} \mathrm{E}_{1,1-\mu}(z) \\
& =\partial_{+}^{(\mu)} \mathrm{e}^{z}, \quad \alpha>0, \beta>0 .
\end{aligned}
$$

Note here that the $\mathrm{E}_{\mu}^{z}$ function for the fractional shift operator $\hat{T}^{\alpha}$ plays the same role as a usual exponent function $\mathrm{e}^{z}$ for the shift operator of the integer order. The list could be supplemented by Wright function, Fox $H$-functions, etc. [18].

\section{References}

[1] M. Kac, Probability and Related Topics in Physical Sciences, Ch. IV (Interscience, New York, 1959).

[2] P. Lévy, The'orie de l'Addition des Variables Aléatoires (Gauthier-Villaws, Paris, 1937).

[3] B.B. Mandelbrot and J.W. van Ness, Fractional Brownian motions, fractional noises and applications, SIAM (Soc. Ind. Appl. Math.) Rev. 70(4), 422-437 (1968).

[4] J. Klafter, A. Blumen, and M.F. Shlesinger, Stochastic pathway to anomalous diffusion, Phys. Rev. A 55(7), 3081-3085 (1987).

[5] G.M. Zaslavsky, Fractional kinetic equation for Hamiltonian chaos, Physica D 76(1-3), 110-122 (1994).

[6] G. Zimbardo, P. Veltri, G. Basile, and S. Principato, Anomalous diffusion and Lévy random walk of magnetic field lines in three dimensional turbulence, Phys. Plasmas 2(7), 2653-2663 (1995).

[7] R.N. Mantega and H.E. Stanley, Scaling behaviour in the dynamics of an economic index, Nature 376(6535), 46-48 (1995).

[8] B.J. West and W. Deering, Fractal physiology for physicists: Lévy statistics, Phys. Rep. 246(1-2), 1-100 (1994).

[9] Fractional Differentiation and its Applications, eds. A. Le Mehauté et al. (Books on Demand, Norderstedt, 2005).

[10] R. Metzler and J. Klafter, The restaurant at the end of the random walk: Recent developments in the description of anomalous transport by fractional dynamics, J. Phys. A 37(31), R161-R208 (2004).

[11] G.M. Zaslavsky, Chaos, fractional kinetics, and anomalous transport, Phys. Rep. 371(6), 461-580 (2002).

[12] E. Lutz, Fractional transport equations for Lévy stable processes, Phys. Rev. Lett. 86(12), 2208-2211 (2001).

[13] N. Laskin, Fractional quantum mechanics, Phys. Rev. E 62 (3), 3135-3145 (2000).

[14] M. Naber, Time fractional Schrödinger equation, J. Math. Phys. 45(8), 3339-3356 (2004).

[15] H. Kleinert, Path Integrals in Quantum Mechanics, Statistics and Polymer Physics (World Scientific, Singapore, 1990).

[16] L.D. Landau and E.M. Lifshitz, Quantum Mechanics, Course of Theoretical Physics, Vol. 1 (Nonrelativistic Theory) (Pergamon, New York, 1965).

[17] A. Marchaud, Sur les dérivées et sur les differences des functions de variables réelles, J. Math. Pures Appl. 6(4), 337-425 (1927).

[18] S.G. Samko, A.A. Kilbas, and O.I. Marichev, Fractional Integrals and Derivatives. Theory and Applications (Gordon and Breach, Amsterdam, 1993). 


\section{VIENMATIS TRUPMENINIS KVANTINIS JUDESIO KIEKIO OPERATORIUS}

\section{P. Miškinis}

Vilniaus Gedimino technikos universitetas, Vilnius, Lietuva

\section{Santrauka}

Kvantinio Lévy (Levi) vyksmo atveju pasiūlyta ermitinio vienmačio judesio kiekio operatoriaus išraiška

$$
\hat{p}=\frac{\hbar l_{0}^{\alpha-1}}{2}\left[\left(-\mathrm{i} \partial_{+}\right)^{\alpha}+\left(\mathrm{i} \tilde{\partial}_{-}\right)^{\alpha}\right]
$$

rastos jo tikrinès funkcijos bei tikrinès vertès

$$
\psi(x, t)=A \mathrm{e}^{\mathrm{i} \kappa x-\mathrm{i} E / \hbar t}, \quad \kappa=\left(\frac{p}{\hbar l_{0}^{\alpha-1}}\right)^{1 / \alpha} .
$$

Išreikštu pavidalu rasta normavimo konstanta

$$
A=\sqrt{\frac{|\kappa|}{2 \pi|p|}} \rightarrow \frac{1}{\sqrt{2 \pi \hbar}}, \quad \text { kai } \quad \alpha \rightarrow 1
$$

ir poslinkio operatoriaus išraiška. Sąryšis tarp judesio kiekio ir banginio skaičiaus

$$
p=\hbar l_{0}^{\alpha-1} \kappa^{\alpha}
$$

apibendrintas vyksmams, turintiems trupmenini Lévy indeksą. 